\title{
Anterior Cingulate and Frontal Lobe White Matter Spectroscopy in Early Childhood of Former Very LBW Premature Infants
}

\author{
JOHN P. PHILLIPS, DAVID RUHL, ERICA MONTAGUE, CHARLES GASPAROVIC, ARVIND CAPRIHAN, ROBIN K. OHLS, \\ RONALD SCHRADER, AND JEAN R. LOWE
}

\author{
Department of Neurology [J.P.P.], Department of Pediatrics [R.K.O., J.R.L.], and Clinical and Translational Science Center [R.S.], \\ University of New Mexico Health Science Center, Albuquerque, New Mexico 87106; The Mind Research Network [D.R., A.C.], \\ Albuquerque, New Mexico 87106; Department of Psychology [E.M., C.G.], University of New Mexico, Albuquerque, New Mexico 87106
}

\begin{abstract}
Neurometabolic sequelae of children born at very LBW (VLBW) are not well characterized in early childhood. Proton magnetic resonance spectroscopy (1H-MRS) and developmental assessments were acquired from children age 18-22 mo (16 VLBW/7 term) and 3-4 y (12 VLBW/8 term) from the anterior cingulate and left frontal periventricular white matter. Metabolites obtained included combined $\mathrm{N}$-acetylaspartylglutamate and $\mathrm{N}$-acetylaspartate (NAA), total choline-containing compounds (Cho), combined glutamate and glutamine (Glx), combined creatine and phosphocreatine $(\mathrm{Cr})$, myoinositol $(\mathrm{mI})$, and the following ratios: NAA/Cr, Cho/Cr, Glx/Cr, mI/Cr, and NAA/ Cho. Significant differences were present only in white matter: at $18-22$ mo, NAA was decreased in VLBW children $(p<0.04)$, and at 3-4 y, VLBW children showed lower $\mathrm{Cr}(p<0.01)$, lower NAA/Cho $(p<0.005)$, higher Glx/Cr $(p<0.02)$, and higher $\mathrm{Cho/Cr}(p<0.005)$. On developmental testing, VLBW children scored lower on language expression $(p<0.05)$ and on the A-not-B test of early executive function $(p<0.01)$ at $18-22$ mo and had lower verbal intelligence quotient (IQ) $(p<0.005)$, performance IQ $(p<0.04)$, and several measures of early executive function including the bear-dragon test $(p<0.004)$, gift delay $(p<0.07)$, and summary categorization score $(p<0.03)$ at 3-4 y. VLBW children may have neurometabolic and developmental abnormalities that persist at least through early childhood. (Pediatr Res 69: 224-229, 2011)
\end{abstract}

$T_{1}$ here are almost 60,000 very LBW (VLBW) infants born in the United States annually (1). Survival has improved in recent decades; however, morbidity is high. Unfortunately, even premature children with normal intelligence quotient (IQ) are at high risk for school failure due to deficits in attention, visuospatial processing, and working memory (2). Furthermore, frequent social problems and poor adaptive skills (3-7) suggest long-term deficits in executive function, which can be difficult to identify in early childhood due to a lack of appropriate assessment measures. Understanding the relationship between prematurity and neurometabolite levels over time may assist in early diagnosis of executive

Received July 22, 2010; accepted October 8, 2010.

Correspondence: John Phillips, M.D., The Mind Research Network, 1101 Yale Boulevard NE, Albuquerque, NM 87106; e-mail: jphillips@mrn.org

Supported by grants from University of New Mexico Clinical and Translational Science Center 1ULRR031977-01, and The Mind Research Network (Department of Energy grant \#DE-FG02-08ER64581) [to J.P.P.] dysfunction and other developmental disorders associated with prematurity.

The pathophysiology of acute brain injury related to prematurity has been characterized (8). However, chronic neurologic sequelae are less well understood, particularly for children without major structural injury. Proton magnetic resonance spectroscopy (1H-MRS) is an in vivo probe of neurometabolism allowing assessment of several metabolites including $\mathrm{N}$-acetylaspartate (NAA), choline-containing compounds (Cho), myoinositol (mI), combined glutamate and glutamine (Glx), and combined creatine and phosphocreatine (Cr). Numerous studies have shown that neurochemical abnormalities observed by MRS are predictive of outcome in term children with encephalopathy $(9-15)$; however, there are few reports using MRS in preterm children. Robertson et al. (16) reported abnormal elevations of the metabolite ratios $\mathrm{mI} / \mathrm{Cr}$ and lactate/Cr in preterm infants with white matter damage when performed at term, although the only study evaluating developmental outcome after prematurity failed to demonstrate a correlation between neonatal 1H-MRS and Bayley motor scores at 18-24 mo of age (17). Two groups have reported $1 \mathrm{H}-\mathrm{MRS}$ in adolescents who were former premature children, showing significant depression of NAA and $\mathrm{Cr}$ in the hippocampus (18) and a trend for lower NAA/Cho, lower NAA/Cr, and higher $\mathrm{Cho} / \mathrm{Cr}$ ratios in left frontal lobe white matter (19). We are not aware of any studies using 1H-MRS in early childhood to evaluate the neurometabolic sequelae of prematurity.

We, therefore, performed a cross-sectional study of development and 1H-MRS in former VLBW children in two age groups: between 18 and 22 mo and between 3 and 4 y of age. Because there may be different effects of prematurity on gray versus white matter, and with of our interest in early executive function, two regions were chosen for analysis: the left frontal white matter and anterior cingulate gyrus. Our goal was to

Abbreviations: 1H-MRS, proton magnetic resonance spectroscopy; BSIDIII, Bayley Scales of Infant Development-III; Cho, total choline-containing compounds; Cr, combined creatine and phosphocreatine; Glx, combined glutamate and glutamine; mI, myoinositol; NAA, combined $N$-acetylaspartylglutamate and $N$-acetylaspartate; PIQ, performance intelligence quotient; TE, echo time; IQ, intelligence quotient; VIQ, verbal intelligence quotient; VLBW, very LBW; WPPSI-III, Wechsler Preschool and Primary Scale of Intelligence-III 
Table 1. Subject demographics and clinical characteristics

\begin{tabular}{|c|c|c|c|c|}
\hline & $\begin{array}{c}18-22 \text { mo preterm } \\
(n=16)\end{array}$ & $\begin{array}{c}18-22 \text { mo control } \\
(n=7)\end{array}$ & $\begin{array}{l}3-4 \text { y preterm } \\
\quad(n=12)\end{array}$ & $\begin{array}{l}3-4 \text { y control } \\
\quad(n=8)\end{array}$ \\
\hline Gestational age (wk, $\pm \mathrm{SD})$ & $28.1 \pm 1.4$ & $39.3 \pm 1.4$ & $27.50 \pm 1.45$ & $36.29 \pm 1.94$ \\
\hline Birth weight $(g, \pm S D)$ & $1069 \pm 192$ & $3104 \pm 171$ & $750 \pm 249.57$ & $2690 \pm 514.47$ \\
\hline Gender (male/female) & $9 / 7$ & $4 / 3$ & $11 / 1$ & $7 / 1$ \\
\hline Age (mo, $\pm \mathrm{SD})$ & $20 \pm 1.5$ & $21 \pm 1.3$ & $37.70 \pm 3.71$ & $39.07 \pm 3.22$ \\
\hline Intraventricular hemorrhage: grade I/grade II/grade III & $5 / 1 / 2$ & 0 & 0 & 0 \\
\hline
\end{tabular}
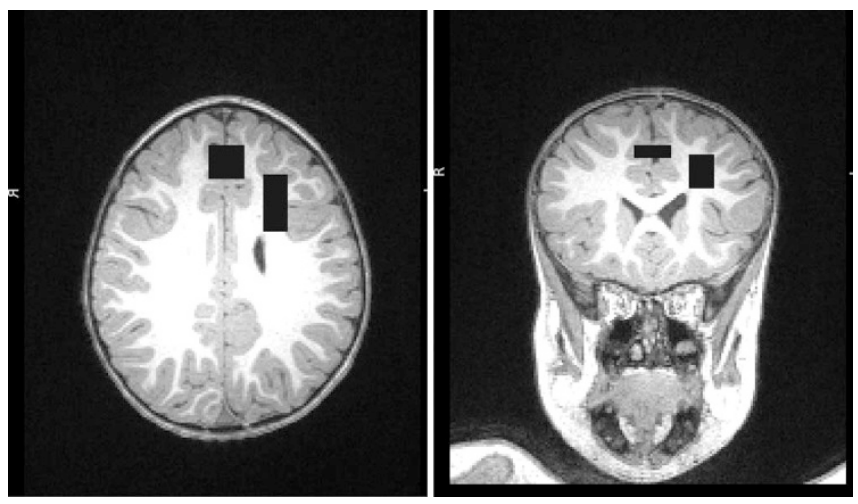

Figure 1. Placement of gray matter anterior cingulate voxel and left frontal white matter voxel.

characterize the regional neurometabolic differences between former VLBW children and healthy term born controls during different early developmental periods. Furthermore, we also sought to identify the effect of prematurity on cognitive development with specific attention to early executive function.

\section{METHODS}

Subjects. This investigation is part of a larger ongoing study of developmental follow-up after prematurity being conducted at the University of New Mexico. Enrollment criteria include birth weight less than $1500 \mathrm{~g}$ with no known history of neonatal stroke, meningitis, grade IV intraventricular hemorrhage, periventricular leukomalacia, hyperbilirubinemia, or other major medical illness or prenatal drug exposure. Children are followed up in a newborn follow-up clinic and those between 18 and 22 mo or 3 and 4 y old were invited to participate. Of 98 total children who qualified for enrollment, 39 enrolled and attempted an MRI scan of which 28 VLBW children did not have excessive movement artifact during spectroscopy acquisition (16 were between 18 and 22 mo old, 12 were between 3 and 4 y old). Control children were healthy without known developmental disorders and were recruited through community advertisements. Thirty controls attempted an MRI scan, and 15 successfully provided spectroscopy datasets ( 7 were $18-22 \mathrm{mo}$, and 8 were $3-4$ y old). The study was approved by the University of New Mexico Human Research Review Committee, and informed consent was obtained from parents of all participants. See Table 1 for participant demographic information.

Developmental assessments. All assessments were conducted at the Mind Research Network by doctoral level graduate students or a senior diagnostician. Parents were allowed to remain with their child during the testing session, which routinely took 1 to $2 \mathrm{~h}$. For children in the 18-22 mo cohort, age was adjusted for prematurity. The younger children completed the Bayley Scales of Infant Development (BSID)-III (20), including the cognitive composite and the language composite, consisting of receptive and expressive language subscales. Early executive functioning was assessed using the A-not-B Task (21). This task involves finding a hidden object beneath one of two cups after a 5- or 12-s delay. Successful completion of this task involves working memory (i.e., remembering where the object is hidden) and response inhibition (i.e., being able to inhibit previous learning when the object changes location) (22). Scoring is based on percent of successful correct responses.

Chronologic age was used for premature children in the 3-4-y-old cohort. These older children completed the Wechsler Preschool and Primary Scale of Intelligence (WPPSI)-III (23), which provides a measure of verbal ability [verbal IQ (VIQ)] and performance-based cognitive ability [performance IQ (PIQ)]. In addition, three measures of executive function were administered:
1) bear/dragon (Simon says) procedures asks the child to either follow or not follow directions from a toy bear and a toy dragon and is scored based on number of times the directions are correctly followed (24),2) gift delay task asks the child not to turn around while the examiner noisily wraps a package for them and is scored by the number of seconds it takes the child to "peek" at the present (25); 3) dimensional change card sort asks the child to variably sort cards by shape or color and is scored based on number of correct sorting responses (DCCS task; 26,27). The bear/dragon and gift delay tasks are measures of response inhibition and self control, and the DCCS task is more an assessment of attentional flexibility.

MRI and MRS data acquisition. Imaging was performed on a Siemens 3-Tesla TrioTIM magnetic resonance scanner using the 12-channel radiofrequency head coil provided with the system (Erlangen, Germany). $\mathrm{T}_{1}$-weighted images were collected in the sagittal plane using a five-echo 3D magnetization prepared rapid gradient echo sequence $[$ repetition time $(\mathrm{TR})=2530$; $\mathrm{TE}$ $($ echo time $)=1.64,3.5,5.36,7.22,9.08$; inversion time $(\mathrm{TI})=1200$; flip angle $=7^{\circ}$; field of view $=256 \times 256 \mathrm{~mm}$; matrix $=256 \times 256,1-\mathrm{mm}$ thick slice, 192 slices; generalized autocalibrating partially parallel acquisitions (GRAPPA) acceleration factor $=2$ ] . These images were used to define two 1 H-MRS voxels (Fig. 1). The first consisted chiefly $(77 \pm 8 \%$ ) of gray matter and was positioned in the bilateral medial frontal cortex, directly superior to the corpus callosum, typically containing the anterior cingulate and portions of the middle frontal and superior frontal gyri. The second voxel was located in the left frontal white matter, as anterior as possible without including significant amounts of gray matter $(79 \pm 13 \% \mathrm{wm})$. For each of these voxels, a point-resolved spectroscopy sequence $(\mathrm{TR} / \mathrm{TE}=1.5 \mathrm{~s} / 40 \mathrm{~ms}$, voxel size $=25 \times 10 \times 15 \mathrm{~mm}$, averages $=192$ ) was collected, using a TE of $40 \mathrm{~ms}$ for improved detection of glutamate (28). An unsuppressed water sequence was collected with 16 averages and otherwise identical parameters to serve as a concentration reference and for eddy current correction in postprocessing. A larger voxel size was used for the first three cases (one control and two VLBW subjects), which measured $20 \times$ $30 \times 30 \mathrm{~mm}$ (see Discussion). Most former premature children were sedated with low dose chloral hydrate at $50 \mathrm{mg} / \mathrm{kg}$ orally.

MRS data analysis. Raw time-domain 1H-MRS data from 4.0 to $1.8 \mathrm{ppm}$ in the spectral dimension were analyzed using LCModel (29) version 6.2, with the unsuppressed water scan as a concentration reference. As a qualityassurance measure, LCModel produces a Cramer-Rao lower bound (CRLB) of the fit to the peak of interest. If this value was greater than $20 \%$ for any major metabolite, the fit was deemed unreliable and excluded from analysis. In addition, if the signal-to-noise ratio (computed by LCModel as the height of the largest peak of fit minus baseline over twice the root mean squared of the residuals of the fit) was below 5, that data were excluded from analysis. Each spectrum was visually inspected for the presence of artifacts or gross fitting errors. By these criteria, one anterior cingulate dataset (from an 18-22 control participant) and three white matter datasets (one 3-4 VLBW, one 18-22 control, and one 3-4 control) were excluded from the analyses presented below. Concentrations were computed for $\mathrm{Cr}$, Cho, mI, NAA, and Glx. To correct for partial-volume and $T_{1}$ and $T_{2}$ relaxation effects, $T_{1}$ weghted images were segmented into gray matter, white matter, and cerebrospinal fluid using Statistical Parametic Mapping version 5 software. Corrected metabolite concentrations were then computed using methods described previously (30).

Statistical analysis. Because of several outliers in the data we used the Mann-Whitney-Wilcoxon test to compare metabolite levels in the two groups; where no outliers were present, independent samples $t$ tests gave comparable results. A value of $p<0.05$ was considered statistically significant.

\section{RESULTS}

Subjects. As noted in Table 1, there were no differences in age at testing between the VLBW and term born children at either age group or were there differences in anthropometric measures including frontal-occipital circumference between 
VLBW and term born children (data not shown). Demographic data such as socioeconomic status and maternal education were similar in all four groups studied.

MRS data. A neuroradiologist reviewed all scans, and no parenchymal abnormalities were noted on high resolution magnetization prepared rapid gradient echo images. Typical anterior cingulate and left frontal white matter voxel placement is shown in Fig. 1. Figure 2 demonstrates representative spectra from the frontal white matter voxel-note discernable peaks present in each subject group for NAA, Cho, Cr, mI, and Glx.

Table 2 summarizes spectroscopy findings including ratio and absolute concentration data for controls and VLBW chil-

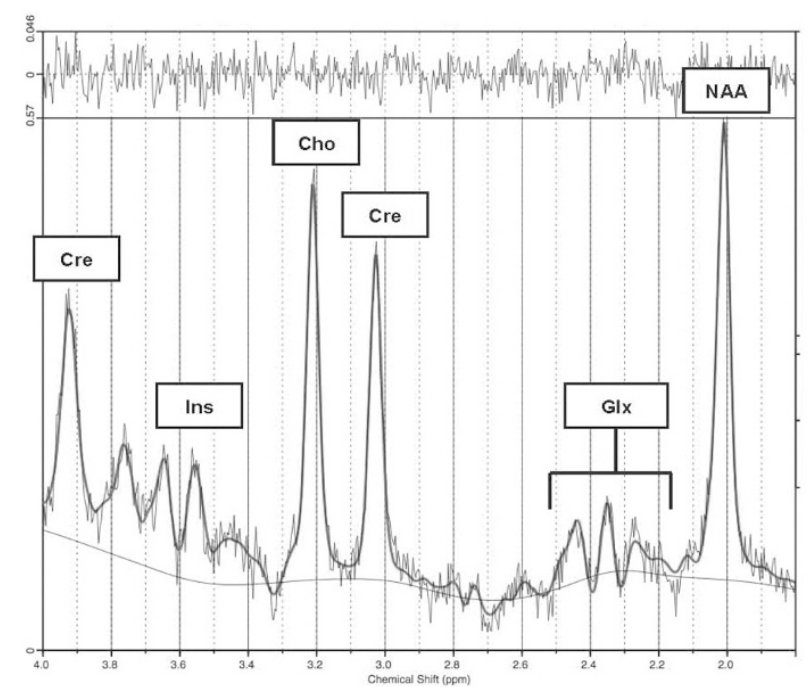

Figure 2. Representative LC model fit to left frontal white matter spectrum from a control participant. dren in both age groups. There were no statistically significant differences between VLBW and control children in the anterior cingulate gyrus at either age. In the left frontal white matter, in 18-22 mo group, NAA was decreased in VLBW children $(p<0.04)$ and, in 3-4 y group, VLBW children had decreased NAA $(p<0.06)$, decreased $\mathrm{Cr}(p<0.01)$, increased $\mathrm{Glx} / \mathrm{Cr}(p<0.02)$, increased $\mathrm{Cho} / \mathrm{Cr}(p<0.005)$, and decreased NAA/Cho $(p<0.005)$; there was a trend for increased Cho in VLBW children at 3-4 y ( $p<0.09$, see Fig. 3 and Table 3).

Developmental data. Significant differences were found between children born VLBW and term at both age groups. At 18-22 mo, term born children scored significantly higher than the VLBW children on the expressive language subscale of the BSID-III $(p<0.041)$ and the A-not-B test $(p<0.008)$. No differences between groups were found on the receptive language subscale or cognitive domain of the BSID-III. At 3-4 y, term born children scored significantly higher on the VIQ from the WPPSI-III $(p<0.004)$ and PIQ $(p<0.036)$. Term born children also performed significantly better on a number of executive function assessments including the bear-dragon test $(p<0.003)$ and the summary categorization score $(p<$ $0.025)$ as well as the gift delay task ( $p<0.068$, see Table 4$)$.

\section{DISCUSSION}

We report the first investigation of former VLBW children in early childhood using 1H-MRS and developmental assessments. Our major finding is that significant metabolic differences are present in the left frontal lobe white matter but not anterior cingulate gyrus of VLBW children compared with term born children. As expected, we also found evidence for impaired development in the VLBW group with significant

Table 2. Single voxel metabolite concentrations

\begin{tabular}{|c|c|c|c|c|c|c|}
\hline Region/metabolite & 18 mo control & 18 mo VLBW & $\begin{array}{c}18 \text { mo } p \\
\text { (VLBW vs control) }\end{array}$ & $3-4$ y control & $3-4$ y VLBW & $\begin{array}{c}3-4 \text { у } p \\
\text { (VLBW vs control) }\end{array}$ \\
\hline \multicolumn{7}{|l|}{ Anterior cingulate ratios } \\
\hline $\mathrm{NAA} / \mathrm{Cr}$ & $1.27 \pm 0.071$ & $1.2 \pm 0.09$ & 0.1148 & $1.21 \pm 0.060$ & $1.23 \pm 0.095$ & 0.7345 \\
\hline $\mathrm{Cho} / \mathrm{Cr}$ & $0.24 \pm 0.021$ & $0.24 \pm 0.027$ & 0.5411 & $0.22 \pm 0.022$ & $0.23 \pm 0.023$ & 0.1813 \\
\hline $\mathrm{MyoI} / \mathrm{Cr}$ & $1.003 \pm 0.136$ & $1.05 \pm 0.11$ & 0.9714 & $0.96 \pm 0.067$ & $0.96 \pm 0.11$ & 0.6784 \\
\hline $\mathrm{Glx} / \mathrm{Cr}$ & $1.65 \pm 0.071$ & $0.167 \pm 0.266$ & 0.4494 & $1.56 \pm 0.14$ & $1.58 \pm 0.12$ & 0.5714 \\
\hline NAA/Cho & $5.28 \pm 0.53$ & $5.10 \pm 0.40$ & 0.971 & $5.59 \pm 0.47$ & $5.37 \pm 0.55$ & 0.3430 \\
\hline \multicolumn{7}{|l|}{ Anterior cingulate concentrations } \\
\hline NAA & $14.15 \pm 0.83$ & $14.17 \pm 1.24$ & 0.9714 & $14.65 \pm 0.892$ & $14.539 \pm 0.767$ & 0.5714 \\
\hline Cho & $2.69 \pm 0.14$ & $2.8 \pm 0.40$ & 0.5411 & $2.63 \pm 0.163$ & $2.728 \pm 0.27$ & 0.5208 \\
\hline MyoI & $11.16 \pm 1.2$ & $12.31 \pm 1.28$ & 0.1335 & $11.58 \pm 1.143$ & $11.422 \pm 1.322$ & 0.9101 \\
\hline Glx & $18.39 \pm 1.27$ & $19.73 \pm 3.76$ & 0.59 & $18.887 \pm 2.495$ & $18.777 \pm 2.28$ & 0.7921 \\
\hline $\mathrm{Cr}$ & $11.19 \pm 0.78$ & $11.78 \pm 0.74$ & 0.098 & $12.123 \pm 0.995$ & $11.903 \pm 1.176$ & 0.6239 \\
\hline \multicolumn{7}{|l|}{ Left anterior white matter ratios } \\
\hline $\mathrm{NAA} / \mathrm{Cr}$ & $1.35 \pm 0.081$ & $1.32 \pm 0.11$ & 0.407 & $1.35 \pm 0.052$ & $1.39 \pm 0.11$ & 0.740 \\
\hline $\mathrm{Cho} / \mathrm{Cr}$ & $0.367 \pm 0.035$ & $0.353 \pm 0.038$ & 0.5162 & $0.35 \pm 0.014$ & $0.42 \pm 0.062$ & 0.0046 \\
\hline $\mathrm{MyoI} / \mathrm{Cr}$ & $0.979 \pm 0.139$ & $1.047 \pm 0.112$ & 0.1545 & $0.84 \pm 0.11$ & $0.83 \pm 0.14$ & 0.9623 \\
\hline $\mathrm{Glx} / \mathrm{Cr}$ & $1.45 \pm 0.148$ & $1.491 \pm 0.291$ & 0.85 & $1.28 \pm 0.13$ & $1.47 \pm 0.11$ & 0.014 \\
\hline NAA/Cho & $3.70 \pm 0.33$ & $3.75 \pm 0.27$ & 0.8577 & $3.83 \pm 0.19$ & $3.38 \pm .74$ & 0.0046 \\
\hline \multicolumn{7}{|c|}{ Left anterior white matter concentrations } \\
\hline NAA & $13.44 \pm 0.48$ & $12.86 \pm 1.13$ & 0.0402 & $14.32 \pm 2.56$ & $12.77 \pm 0.77$ & 0.0553 \\
\hline Cho & $3.65 \pm 0.35$ & $3.44 \pm 0.384$ & 0.1148 & $3.73 \pm 0.56$ & $3.86 \pm 0.46$ & 0.0878 \\
\hline MyoI & $7.83 \pm 1.298$ & $8.63 \pm 1.423$ & 0.3668 & $8.78 \pm 1.20$ & $7.69 \pm 1.55$ & 0.1932 \\
\hline Glx & $14.44 \pm 1.31$ & $14.27 \pm 2.64$ & 0.5187 & $13.47 \pm 1.39$ & $13.60 \pm 1.43$ & 0.7396 \\
\hline $\mathrm{Cr}$ & $9.94 \pm 0.294$ & $9.75 \pm 1.01$ & 0.2308 & $10.57 \pm 1.59$ & $9.23 \pm 0.59$ & 0.0097 \\
\hline
\end{tabular}



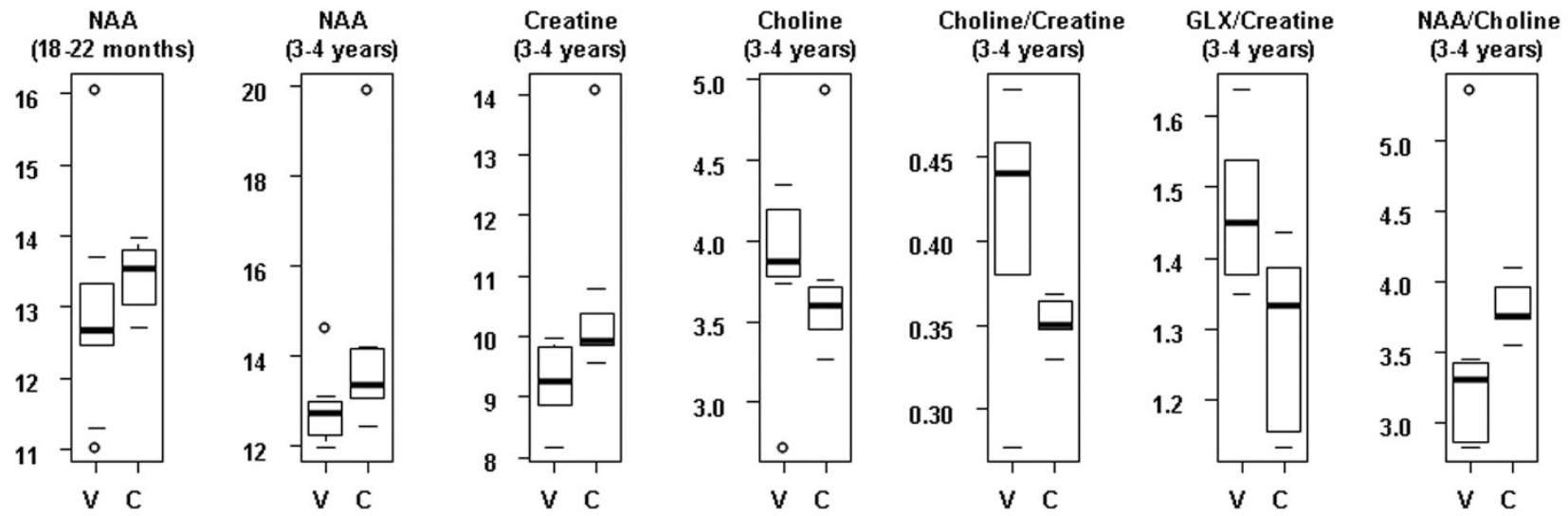

Figure 3. Boxplots of metabolic data showing group differences for the left frontal lobe white matter demonstrate lower NAA in former VLBW children compared with controls at $18-22$ mo $(p<0.040)$. At 3-4 y, there was also lower NAA $(p<0.055)$, lower creatine $(p<0.010)$, increased choline $(p<0.088)$, increased choline/creatine $(p<0.005)$, increased Glx/creatine $(p<0.014)$, and decreased NAA/Cho $(p<0.005)$. Metabolite concentrations are in millimoles per kilogram tissue water are on the $y$ axis, and $x$ axis represents VLBW (V) or term control (C) subject groups.

Table 3. The p-values and confidence intervals for selected neurometabolites as noted in boxplots (Fig. 3)

\begin{tabular}{|c|c|c|c|}
\hline & \multicolumn{2}{|c|}{ Confidence bounds } & \multirow[b]{2}{*}{$p$} \\
\hline & Lower & Upper & \\
\hline NAA (18-22 mo) & -1.372 & -0.045 & 0.040 \\
\hline NAA (3-4 y) & -1.975 & 0.051 & 0.055 \\
\hline Creatine (3-4 y) & -1.810 & -0.120 & 0.010 \\
\hline Choline (3-4 y) & -0.581 & 0.636 & 0.088 \\
\hline Cho/Cre (3-4 y) & 0.029 & 0.112 & 0.005 \\
\hline Glx/Cre (3-4 y) & 0.031 & 0.304 & 0.014 \\
\hline NAA/Cho (3-4 y) & -0.913 & -0.314 & 0.005 \\
\hline
\end{tabular}

Table 4. Developmental assessments

\begin{tabular}{lcc}
\hline & $\begin{array}{c}\text { Control, } \\
\text { score } \pm \text { SD }\end{array}$ & $\begin{array}{c}\text { VLBW, } \\
\text { score } \pm \text { SD }\end{array}$ \\
\hline 18-22 mo assessments & & \\
BSID-III & & \\
$\quad$ Language Domain & $94.86 \pm 7.88$ & $86.63 \pm 11.78$ \\
$\quad$ Expressive Language Scaled Score* & $9.00 \pm 1.73$ & $7.06 \pm 1.98$ \\
$\quad$ Receptive Language Scaled Score & $9.29 \pm 1.89$ & $8.4 \pm 2.6$ \\
$\quad$ Cognitive Domain & $98.57 \pm 5.56$ & $93.75 \pm 12.45$ \\
A not B* & $0.95 \pm 0.08$ & $0.63 \pm 0.30$ \\
3-4 y assessments & & \\
$\quad$ WPPSI-III & $108.75 \pm 10.61$ & $90.58 \pm 12.67$ \\
$\quad$ Verbal IQ* & $102 \pm 10.18$ & $90.83 \pm 12.39$ \\
$\quad$ Performance IQ & $26.63 \pm 11.38$ & $8.42 \pm 9.00$ \\
Bear Dragon Test* & $39.88 \pm 21.59$ & $20.92 \pm 18.94$ \\
Gift Delay Task* & $3.13 \pm 0.76$ & $2.12 \pm 1.01$ \\
DCCS Summary Categorization* & \\
\hline
\end{tabular}

* Differences between VLBW and control children significant at $p<0.05$. BSID-III Domain scores are Standard Scores with mean of 100, SD of 15; BSID-III subscales are Scaled Scores with mean of 10, SD of 3; A not B score is percentage of items passed; WPPSI-III scores are Standard Scores with mean of 100, SD of 15; Bear Dragon Test score ranges from 0 to 33; Gift Delay score is in seconds; DCCS Summary Categorization is a summary score across four tasks ranging from 0 to 4 .

effects on expressive language and emerging executive function. Taken together, these results suggest that prematurity has neurometabolic and developmental effects that are present in early childhood.

Although there are no reports using 1H-MRS to characterize the neurometabolic effect of prematurity in early child- hood, there are several reports of $1 \mathrm{H}-\mathrm{MRS}$ in adolescents who were born prematurely. In a group of 18 former VLBW children who underwent MRS at $15 \mathrm{y}$ of age, Bathen et al. (19) found no significant difference in the left frontal white matter compared with controls, although there was a consistent trend for the VLBW group to have lower NAA/Cho, lower NAA/Cr, and higher $\mathrm{Cho} / \mathrm{Cr}$ ratios. These subjects were included in a larger study by Vangberg et al. (31) who reported diffusion tensor imaging in 34 former VLBW children at $15 \mathrm{y}$ of age and found lower fractional anisotropy in the left frontal white matter compared with controls. Thus, the diffusion tensor imaging and $1 \mathrm{H}-\mathrm{MRS}$ data from this cohort suggests that prematurity may cause long-term disruption of white matter tracts and possible neurometabolic changes. In a different group of subjects, $1 \mathrm{H}-\mathrm{MRS}$ of the hippocampus at a mean age of $15 \mathrm{y}$ in former premature children showed significant decreases in absolute concentrations of NAA and $\mathrm{Cr}$ as well as ratios of NAA/Cho and $\mathrm{Cr} / \mathrm{Cho}$ (18). However, it is not clear how prematurity may affect the neurometabolic profile of younger children.

Our study is the first that uses 1H-MRS to examine former VLBW children in early childhood. Children born at term are included because MRS normative data are not available, and computed metabolite concentrations may vary based on the data acquisition and analysis methods used (30). We found no significant differences between VLBW and term children in the anterior cingulate gyrus at either age. However VLBW children had important age-related differences in white matter metabolites; at 18-22 mo, NAA was decreased in former VLBW children in the left frontal lobe white matter $(p<$ $0.04)$, whereas at 3-4 y, there were decreases in white matter $\mathrm{Cr}(p<0.01)$ and NAA $(p<0.06)$, increased Cho $(p<0.09)$, elevated ratios of $\mathrm{Glx} / \mathrm{Cr}(p<0.02)$, and $\mathrm{Cho} / \mathrm{Cr}(p<0.005)$ and decreased NAA/Cho $(p<0.005)$.

The metabolite differences we found in VLBW children are consistent with 1H-MRS reports in other disease groups. NAA in the white matter was decreased in our VLBW children at $18-22 \mathrm{mo}$ and at $3-4 \mathrm{y}$, which is similar to the trend of lower NAA in adolescents who were former VLBW (19). Depressed 
NAA has been reported in other diseases such as neonatal hypoxic-ischemic encephalopathy (15). However, NAA seems to be related to neuronal integrity and function, not just neuronal mass (32), and decreased NAA may be partially reversible, as shown in diseases such as traumatic brain injury (33) or multiple sclerosis (34). Longitudinal studies will be required to characterize how NAA may change over time after premature delivery and to determine whether there is an age at which NAA may help identify children at risk for later neurocognitive impairment.

Further, white matter differences were present in our 3-4 y VLBW children, including depressed $\mathrm{Cr}$ and elevated Cho, as well as elevated ratios of $\mathrm{Cho} / \mathrm{Cr}$ and $\mathrm{Glx} / \mathrm{Cr}$ and depressed NAA/Cho. The finding of elevated Cho has been reported in white matter diseases such as multiple sclerosis (35), traumatic brain injury (36), and Krabbe leukodystrophy (37). The Cho signal of 1H MRS primarily arises from phosphocholine and glycerophosphocholine, compounds that are involved in the synthesis and breakdown of phosphatidylcholine, a major phospholipid component of myelin and cell membranes (38). There is a decrease in $1 \mathrm{H}-\mathrm{MRS}$-detected Cho in the first $2 \mathrm{y}$ of life, roughly corresponding to normal white matter myelination. With pathology involving demyelination and membrane breakdown, the Cho signal rises. The known cerebral white matter injury associated with prematurity in some children (8) suggests a possible mechanism for membrane injury causing elevated Cho.

Similarly, although total $\mathrm{Cr}$ is often used as an internal standard when reporting $1 \mathrm{H}-\mathrm{MRS}$ as ratio data, it can be altered in some disorders such as traumatic brain injury (36), which may be consistent with the central role of $\mathrm{Cr}$ and phosphocreatine in normal brain energy metabolism in both neurons and glial cells (39). Our finding of decreased $\mathrm{Cr}$ is consistent with a prior report of former VLBW children as adolescents who had decreased Cr on MRS of the hippocampus (18) and suggests that even in subjects without overt structural lesions, $\mathrm{Cr}$ might be a marker of cellular injury and disrupted energy metabolism associated with prematurity. However, this also complicates interpretation of ratio data, which, in our subjects, showed significant elevations of $\mathrm{Cho} / \mathrm{Cr}$ and $\mathrm{Glx} / \mathrm{Cr}$ that could be largely driven by the decreased $\mathrm{Cr}$ rather than solely from increases in Glx or Cho.

The only spectroscopic differences between VLBW and control children were identified in the white matter voxel. This striking variance may be due to the known white matter vulnerability to injury associated with prematurity, which in many cases can be difficult to identify on standard neuroimaging (8). It is also possible that the lack of group differences in the anterior cingulate voxel reflects regional vulnerability, as prior work suggests thalamus and basal ganglia neurometabolites may be significantly affected in term children with hypoxic injury $(12,15)$. Further studies are needed to clarify this finding, which may have implications for early diagnosis of injury after premature delivery.

Developmental test results demonstrated group differences between control and VLBW children, following a similar trend in both age groups. In the 18-22 mo and the 3-4 y age cohorts, the only domains where term born children performed significantly better than VLBW children were measures of expressive language and executive function. In addition, in the 3-4 y age group, term children also did significantly better on performance-based cognitive tasks, suggesting that general cognition may be affected in the VLBW group. The lack of deficits in other areas of general cognition for the 18-22 mo group was unexpected, as more global cognitive impairments have been reported (42). However, it is possible that the constellation of cognitive deficits resulting from premature birth changes over the course of childhood, with some abilities recovering at the expense of others (43). It is also possible that there was selection bias, as only children who were able to cooperate with the MRI scan were included in this study. Longitudinal studies with larger sample sizes will be required to determine how cognitive deficits associated with prematurity change over time.

Our finding that healthy term born children perform better on measures of executive function is less surprising. Executive function deficits are consistently reported in the literature on VLBW children, emerging as early as 8 mo (44) and persisting through preschool into middle childhood (45). These deficits predict later educational outcome (3). Furthermore, deficits in executive function may underlie the significant social problems and poor adaptive skills frequently experienced by former VLBW children as they grow (3-7).

There are several shortcomings of this study. As a crosssectional investigation, we cannot use $1 \mathrm{H}-\mathrm{MRS}$ to predict developmental outcome. Also, our low subject numbers limit statistical power, and we, therefore, could not perform correlations between the many behavioral assessments and $1 \mathrm{H}-$ MRS findings. In addition, the first three subjects had larger voxel sizes. However, because the larger voxels did not include fractions of gray and white matter that differed from the smaller voxels and, furthermore, were not exclusively in one group, these datasets were included. A final shortcoming is that although this study excluded children with any history of major medical illness, grade IV intraventricular hemorrhage or periventricular leukomalacia, our structural scans did not include $\mathrm{T}_{2}$ or fluid attenuated inversion recovery sequences; therefore, it is possible that gliosis was missed. Still, all high-resolution $\mathrm{T}_{1}$-weighted images were evaluated by a neuroradiologist, and no lesions suggestive of periventricular leukomalacia were noted, nor was periventricular leukomalacia present on the routine neonatal head ultrasound examinations.

Clearly, longitudinal studies are required to determine whether there is a role for $1 \mathrm{H}-\mathrm{MRS}$ in early detection of specific developmental problems after prematurity. Matched controls will be necessary in such studies, and because of ethical issues that prohibit sedating healthy term born children, these studies will require multidisciplinary teams experienced with pediatric imaging and developmental assessments. However, these studies are worth pursuing as a better understanding of brain-behavioral relationships in VLBW children may ultimately lead to earlier and more effective interventions. 
Acknowledgments. We gratefully acknowledge the critical role played by the following people: Susanne Duvall, Lynette Silva, Diana South, Cathy Smith, Judith Segall, Joy Van Meter, Becky Montman, Carol Hartenberger, Mashid Rhoohi, Conra Backstrom-Lacy, and Dr. Joyce Phillips.

\section{REFERENCES}

1. Martin JA, Kung HC, Mathews TJ, Hoyert DL, Strobino DM, Guyer B, Sutton SR 2008 annual summary of vital statistics: 2006. Pediatrics 121:788-801

2. Vicari S, Caravale B, Carlesimo GA 2004 Spatial working memory deficits in children at ages 3-4 who were low birth weight, preterm infants. Neuropsychology 18:673-678

3. Grunau RE, Whitfield MF, Fay TB 2004 Psychosocial and academic characteristics of extremely low birth weight $(<$ or $=800 \mathrm{~g})$ adolescents who are free of major impairment compared with term-born control subjects. Pediatrics 114:e725-e732

4. Nadeau L, Boivin M, Tessier R, Lefebvre F, Robaey P 2001 Mediators of behavioral problems in 7-year-old children born after 24 to 28 weeks of gestation. J Dev Behav Pediatr 22:1-10

5. Rickards AL, Kelly EA, Doyle LW, Callanan C 2001 Cognition, academic progress, behavior and self-concept at 14 years of very low birth weight children. J Dev Behav Pediatr 22:11-18

6. Anderson PJ, Doyle LW 2004 Executive functioning in school-aged children who were born very preterm or with extremely low birth weight in the 1990s. Pediatrics 114:50-57

7. Edgin JO, Inder TE, Anderson PJ, Hood KM, Clark CA, Woodward LJ 2008 Executive functioning in preschool children born very preterm: relationship with early white matter pathology. J Int Neuropsychol Soc 14:90-101

8. Volpe JJ 2009 Brain injury in premature infants: a complex amalgam of destructive and developmental disturbances. Lancet Neurol 8:110-124

9. Khong PL, Tse C, Wong I, Lam BC, Cheung PT, Goh WH, Kwong NS, Ooi GC 2004 Diffusion-weighted imaging and proton magnetic resonance spectroscopy in perinatal hypoxic-ischemic encephalopathy: association with neuromotor outcome at 18 months of age. J Child Neurol 19:872-881

10. Kadri M, Shu S, Holshouser B, Deming D, Hopper A, Peverini R, Ashwal S 2003 Proton magnetic resonance spectroscopy improves outcome prediction in perinatal CNS insults. J Perinatol 23:181-185

11. Barkovich AJ, Miller SP, Bartha A, Newton N, Hamrick SE, Mukherjee P, Glenn OA, Xu D, Partridge JC, Ferriero DM, Vigneron DB 2006 MR imaging, MR spectroscopy, and diffusion tensor imaging of sequential studies in neonates with encephalopathy. AJNR Am J Neuroradiol 27:533-547

12. Miller SP, Newton N, Ferriero DM, Partridge JC, Glidden DV, Barnwell A, Chuang NA, Vigneron DB, Barkovich AJ 2002 Predictors of 30-month outcome after perinatal depression: role of proton MRS and socioeconomic factors. Pediatr Res 52:71-77

13. Shu SK, Ashwal S, Holshouser BA, Nystrom G, Hinshaw DB 1997 Prognostic value of 1H-MRS in perinatal CNS insults. Pediatr Neurol 17:309-318

14. Groenendaal F, Grond J, Eken P, Haastert C, Rademaker KJ, Toet MC, deVries LS 1997 Early cerebral proton MRS and neurodevelopmental outcome in infants with cystic leukomalacia. Dev Med Child Neurol 39:373-379

15. Cheong JL, Cady EB, Penrice J, Wyatt JS, Cox IJ, Robertson NJ 2006 Proton MR spectroscopy in neonates with perinatal cerebral hypoxic-ischemic injury: metabolite peak-area ratios, relaxation times, and absolute concentrations. AJNR Am J Neuroradiol 27:1546-1554

16. Robertson NJ, Kuint J, Counsell TJ, Ruterford TA, Coutts A, Cox IJ, Edwards AD 2000 Characterization of cerebral white matter damage in preterm infants using $1 \mathrm{H}$ and 31P magnetic resonance spectroscopy. J Cereb Blood Flow Metab 20:14461456

17. Augustine EM, Spielman DM, Barnes PD, Sutcliffe TL, Dermon JD, Mirmiran M, Clayton DB, Ariagno RL 2008 Can magnetic resonance spectroscopy predict neurodevelopmental outcome in very low birth weight preterm infants? J Perinatol 28:611-618

18. Gimenez M, Soria-Pastor SS, Junque C, Caldu X, Narberhaus A, Botet F, Bargello N, Falcon C, Mercader J 2008 Proton magnetic resonance spectroscopy reveals medial temporal metabolic abnormalities in adolescents with history of preterm birth. Pediatr Res 64:572-577
19. Bathen TF, Sjobakk T, Skranes J, Brubakk A, Vik T, Martinussen M, Myhr GE, Gribbestad IS, Axelson D 2006 Cerebral metabolite differences in adolescents with low birth weight: assessment with in vivo proton MR spectroscopy. Pediatr Radiol 36:802-809

20. Bayley N 2005 Bayley Scales of Infant Development Manual. 3rd ed. The Psychological Corporation, San Antonio, TX

21. Diamond A 1985 Development of the ability to use recall to guide action, as indicated by infants' performance on AB. Child Dev 56:868-883

22. Espy KA, Kaufmann PM, McDiarmid MD, Glisky ML 1999 Executive functioning in preschool children: performance on A-not-B and other delayed response format tasks. Brain Cogn 41:178-199

23. Wechsler D 2002 Manual for Wechsler Preschool and Primary Scale of IntelligenceThird Edition (WPPSI-III). The Psychological Corporation, San Antonio, TX

24. Reed MA, Pien DL, Rothbart MK 1984 Inhibitory self-control in preschool children. Merrill Palmer Q 30:131-147

25. Kochanska G, Murray KT, Jacques TY, Koenig AL, Vandegeest KA 1996 Inhibitory control in young children and its role in emerging internalization. Child Dev 67:490-507

26. Frye D, Zelazo PD, Palfai T 1995 Theory of mind and rule-based reasoning. Cogn Dev 10:483-527

27. Zelazo PD, Reznick JS, Pinon DE 1995 Response control and the execution of verbal rules. Dev Psychol 31:508-517

28. Mullins PG, Chen H, Xu J, Caprihan A, Gasparovic C 2008 Comparative reliability of proton spectroscopy techniques designed to improved detection of J-coupled metabolites. Magn Reson Med 60:964-969

29. Provencher SW 2001 Automatic quantitation of localized in vivo $1 \mathrm{H}$ spectra with LCModel. NMR Biomed 14:260-264

30. Gasparovic C, Song T, Devier D, Bockholt HJ, Caprihan A, Mullins PG, Posse S, Jung R, Morrison L 2006 Use of tissue water as a concentration reference for proton spectroscopic imaging. Magn Reson Med 55:1219-1226

31. Vangberg TR, Skranes J, Dale AM, Martinussen M, Brubakk AM, Haraldseth O 2006 Changes in white matter diffusion anisotropy in adolescents born prematurely. Neuroimage 32:1538-1548

32. Moffett JR, Ross B, Arun P, Madhavarao CN, Namboodiri AM 2007 N acteylaspartate in the CNS; from neurodiagnostics to neurobiology. Prog Neurobiol 81:89-131

33. Brooks WM, Stidley CA, Petropoulos H, Jung RE, Weers DC, Friedman SD, Barlow MA, Sibbitt WL Jr, Yeo RA 2000 Metabolic and cognitive response to human traumatic brain injury: a quantitative proton magnetic resonance study. J Neurotrauma 17:629-640

34. Khan O, Shen Y, Bao F, Caon C, Tselis A, Latif Z, Zak I 2008 Long-term study of brain 1H-MRS study in multiple sclerosis: effect of glatiramer acetate therapy on axonal metabolic function and feasibility of long-term H-MRS monitoring in multiple sclerosis. J Neuroimaging 18:314-319

35. Bitsch A, Bruhn H, Vougioukas V, Stringaris A, Lassman H, Frahm J, Bruck W 1999 Inflammatory CNS demyelination: histopathologic correlation with in vivo quantitative proton MR spectroscopy. AJNR Am J Neuroradiol 20:1619-1627

36. Gasparovic C, Yeo R, Mannell M, Ling J, Elgie R, Phillips JP, Doezema D, Mayer A 2009 Neurometabolite concentrations in gray and white matter in mild traumatic brain injury: a H-magnetic resonance spectroscopy study. J Neurotrauma 26:16351643

37. Brockmann K, Dechent P, Wilken B, Rusch O, Frahm J, Hanefeld F 2003 Proton MRS profile of cerebral metabolic abnormalities in Krabbe disease. Neurology 60:819-825

38. Panigrahy A, Borzage M, Bluml S 2010 Basic principles and concepts underlying recent advances in magnetic resonance imaging of the developing brain. Semin Perinatol 34:3-19

39. Wyss M, Kaddurah-Daouk R 2000 Creatine and creatine metabolism. Physiol Rev 80:1107-1213

40. Deleted in proof

41. Deleted in proof

42. Bhutta AT, Cleves MA, Casey PH, Cradock MM, Anand KJ 2002 Cognitive and behavioral outcomes of school-aged children who were born preterm: a metaanalysis. JAMA 288:728-737

43. Luciana M 2003 Cognitive development in children born preterm: implications for theories of brain plasticity following early injury. Dev Psychopathol 15:1017-1047

44. Sun J, Mohay H, O'Callaghan M 2009 A comparison of executive function in very preterm and term infants at 8 months corrected age. Early Hum Dev 85:225-230

45. Espy KA, Stalets MM, McDiarmid MM, Senn TE, Cwik MF, Hamby A 2002 Executive functions in preschool children born preterm: application of cognitive neuroscience paradigms. Child Neuropsychol 8:83-92 